\title{
Quantifying the relationship between sequence and three-dimensional structure conservation in RNA
}

Emidio Capriotti ${ }^{1,2}$ and Marc A Marti-Renom*1

\begin{abstract}
Background: In recent years, the number of available RNA structures has rapidly grown reflecting the increased interest on RNA biology. Similarly to the studies carried out two decades ago for proteins, which gave the fundamental grounds for developing comparative protein structure prediction methods, we are now able to quantify the relationship between sequence and structure conservation in RNA.

Results: Here we introduce an all-against-all sequence- and three-dimensional (3D) structure-based comparison of a representative set of RNA structures, which have allowed us to quantitatively confirm that: (i) there is a measurable relationship between sequence and structure conservation that weakens for alignments resulting in below $60 \%$ sequence identity, (ii) evolution tends to conserve more RNA structure than sequence, and (iii) there is a twilight zone for RNA homology detection.

Discussion: The computational analysis here presented quantitatively describes the relationship between sequence and structure for RNA molecules and defines a twilight zone region for detecting RNA homology. Our work could represent the theoretical basis and limitations for future developments in comparative RNA 3D structure prediction.
\end{abstract}

\section{Background}

The view of RNA as a simple information transfer molecule has been challenged since the discovery of ribozymes, a class of RNA with enzyme-like functions [13]. RNA molecules are now known to carry a large repertory of biological functions such as transfer of information, enzymatic catalysis and regulation of cellular processes [4]. Similar to proteins, functional RNA molecules fold into specific three-dimensional conformations essential for performing their biological activity. Despite advances in characterizing the folding and unfolding of RNA molecules [5-8] and the significant increase of RNA structures deposited in the Protein Data Bank (PDB) [9], our knowledge of the atomic mechanism by which RNA molecules adopt their biological active structures is still limited [10]. Nonetheless, it is common knowledge that RNA 3D structure is more conserved than RNA sequence and that such principle could be used for comparative

\footnotetext{
* Correspondence: mmarti@cipf.es
}

1 Structural Genomics Unit, Bioinformatics and Genomics Department, Centro de Investigación Príncipe Felipe, Valencia, Spain

Full list of author information is available at the end of the article
RNA structure prediction in a similar way it is done for proteins [11]. It was back in the eighties when Chothia and Lesk first quantified such evolutionary relationship for proteins [12-14]. Their seminal works on the relationship between protein sequence and structure conservation provided the theoretical grounds for many computational approaches in comparative protein structure and function prediction $[11,15]$. Their work concluded that the overall structural changes between two homologous proteins were proportional to their sequence differences. It was then estimated that homologous proteins aligning with less than $20 \%$ sequence identity could have large structural differences [14]. Such findings were later confirmed and expanded by several other studies [16-20].

For RNA, the axiom of "function is more conserved than structure and structure is more conserved than sequence" has been adopted since the end of the sixties [21] and even reinforced with the analysis of newly determined large RNA containing complexes such as the ribosome [22-29]. The wealth of new structures has prompted the development of computational methods 
for classifying RNA molecules [30-34], describing their structural features [35-37] assigning their functions $[34,38]$ comparing their structures [39-41] and predicting their structures [42-45]. For example, the relationship between sequence and structure in RNA molecules has previously been characterized for the RNA ribose zipper [28], the C1 region of the env HIV1 gene [46] and RNA loop regions $[47,48]$. A new method that relies on secondary structure information to align homolog RNA sequences was also recently developed [49]. Finally, with the aim of characterizing RNA structure diversity, Abraham and co-workers recently studied the RNA conservation at three levels: primary, secondary, and tertiary structure. The work resulted in the DARTS database [34], which constitutes a new classification of RNA structure after the SCOR database [31]. However, to date no general large-scale study has systematically addressed the quantitative analysis of the relationship between sequence and structure conservation in RNA molecules. The work here introduced aims to address such gap by performing an all-against-all comparison of 451 nonidentical (that is, $100 \%$ sequence identity) RNA structures from the PDB. The resulting analysis confirms in a general and quantitative manner the relationship between sequence and structure conservation in RNA molecules as well as allows the definition of a "twilight zone" for RNA homology detection.

We begin this article by describing the results of an allagainst-all comparison of a non-identical RNA structure set (Results). Next, we discuss the impact that our findings could have in the development of comparative approaches for RNA structure prediction (Discussion). Finally, we end by detailing the methodology used for aligning and assessing RNA alignments (Materials and Methods).

\section{Results}

\section{Accurate pair-wise alignments of RNA structures}

All pair-wise RNA structure alignments were generated by the SARA program [41] using the 114 non-identical RNA structures in the HA-RNA09 dataset. The SARA program was run with default parameters and selecting only based-paired regions of the structures (Methods). HA-RNA09 included 589 pair-wise alignments between 39 tRNA (34.2\%), 21 23S Ribosomal RNA (18.4\%), 14 riboswitches (12.3\%), 11 Ribozymes (9.6\%), $105 \mathrm{~S}$ Ribosomal RNA (8.8\%), 9 16S Ribosomal RNA (7.9\%) an 10 other RNA structures $(8,8 \%)$. Such alignments superposed with PSI values (i.e., percentage of $\mathrm{C}^{\prime}$ atoms within $4.0 \AA$ RMSD) higher than $60 \%$ and only 3 alignments resulted in less than $75 \%$ PSI. The average PSI value for the 589 pair-wise alignments was $90.4 \%$, which corresponded to a core RNA size ranging from $\sim 50$ to $\sim 2,800$ nucleotides. The superimposition of the Staphylo- coccus phage group I ribozyme $(1 \mathrm{y} 0 \mathrm{q}$ PDB identifier, chain A) and a fragment of the synthetic construct group I Intron (1u6b PDB identifier, chain B) resulted in $60.9 \%$ aligned $\mathrm{C} 3$ ' atoms, which corresponded to a structural core of 120 nucleotides (Figure 1A). About $48 \%$ of the pair-wise alignments in the HA-RNA09 dataset aligned two tRNA molecules. The alignment of a tRNA(Leu) of Pyrococcus horikoshii ( $\underline{\mathrm{wz} 2} \mathrm{PDB}$ identifier, chain C) and the tRNA(Met) of Acuifex aeolicus (2ct8 PDB identifier, chain C) resulted in 65 core nucleotides within $1.9 \AA$ RMSD (Figure 1B). About 14\% of the alignments in the HA-RNA09 dataset superposed a pair RNA structures that did not include either a tRNA or a ribosomal RNA. The alignment of two synthetic constructs P4-P6 RNA ribozyme domains $(\underline{118 \mathrm{v}}$ and $\underline{2 \mathrm{r} 8 \mathrm{~s}} \mathrm{PDB}$ identifiers, chains $A$ and $R$, respectively) resulted in 134 core nucleotides within $1.8 \AA$ RMSD (Figure 1C). Finally, 38\% of the alignments in the HA-RNA09 dataset corresponded to alignments between ribosomal RNA molecules. The alignment of $23 \mathrm{~S}$ ribosomal RNA of Haloarcula marismortui (3cce PDB identifier, chain 0) and 23S ribosomal RNA of Thermus thermophilus (3d5b PDB identifier, chain A) resulted in 2,347 core nucleotides within $1.7 \AA$ RMSD (Figure 1D).

\section{The relationship between sequence and structure conservation in RNA}

All the alignments in the HA-RNA09 set superposed a medium to large structurally conserved RNA core. The structure diversity between the selected pairs of RNA structure cores had a measurable relationship to the sequence diversity, which best fitted an exponential decay curve with a 0.67 correlation coefficient (Figure 2A). Similar to proteins, the structure identity decreased with the decrease of the sequence identity and the structure of the core was more conserved than its sequence. The median of the percentage of sequence and structure identity for the 589 alignments in the HA-RNA09 set were $52.3 \%$ and $89.0 \%$, respectively. Sequence identity ranged from 29.2 to $100.0 \%$, with $95 \%$ of the alignments with PID (i.e., percentage of sequence identity) higher than $34.2 \%$. Structure identity ranged from 60.9 to $100.0 \%$, with $95 \%$ of the alignments with PSI higher than $81.2 \%$. The same trend is observed by comparing sequence conservation (PID) with structure diversity (structure core RMSD) (Figure 2B). The relationship between RMSD and percentage of sequence identity fits an exponential curve with a 0.77 correlation coefficient. It is important to note that the pair-wise structure alignments appeared to cluster into two groups around sequence identity ranges of $30-60 \%$ and $90-100 \%$. The correlation coefficient between RMSD and sequence identity in the two groups was $0.34(\mathrm{p}=$ $\left.8.7 \cdot 10^{-11}\right)$ for $30-60 \%$ and $0.63\left(\mathrm{p}=3.8 \cdot 10^{-15}\right)$ for $90-100 \%$, which indicates that as sequence identity decreases, the 
A Staphylococcus phage group I ribozyme (1y@q:A) Synthetic I Intron fragment (1u6b:B)

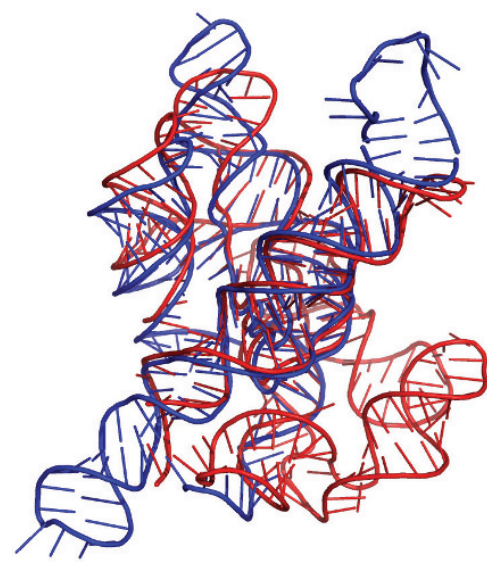

Aligned nucleotides:

120

RMSD :

Sequence Identity:

Secondary Structure Identity:

$1.8 \AA$

$34 . \odot \%$

$52.1 \%$

Structure Identity:

$60.9 \%$

Sequence - ln ( $p$-value):

Secondary structure - ln(p-value):

$\begin{array}{ll}\text { Structure - ln (p-value }): & 15.6\end{array}$

Mean - $\ln (p-v a l u e)$ :

14.7

C Synthetic P4-P6 RNA ribozyme (1l8v:A) Synthetic P4-P6 RNA ribozyme (2r8s:R)

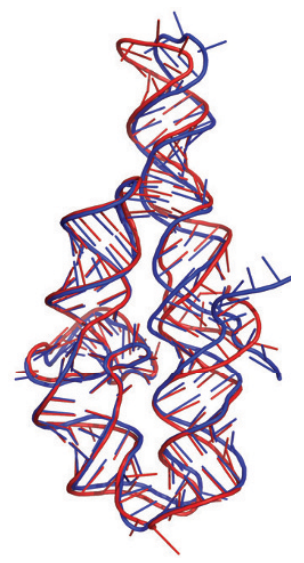

Aligned nucleotides:

134

RMSD :

Sequence Identity:

$1.8 \AA$

$80.9 \%$

Secondary Structure Identity: $\quad 81.0 \%$

Structure Identity:

Sequence - ln (p-value):

$81.0 \%$

Secondary structure - ln ( $p$-value $): 17.1$

Structure - ln ( $p$-value):

19.4

Mean - In (p-value):

24.5
$\mathrm{B}$

Pyrococcus horikoshii tRNA(Leu) (1wz2:C) Acuifex aeolicus tRNA(Met) (2ct8:C)

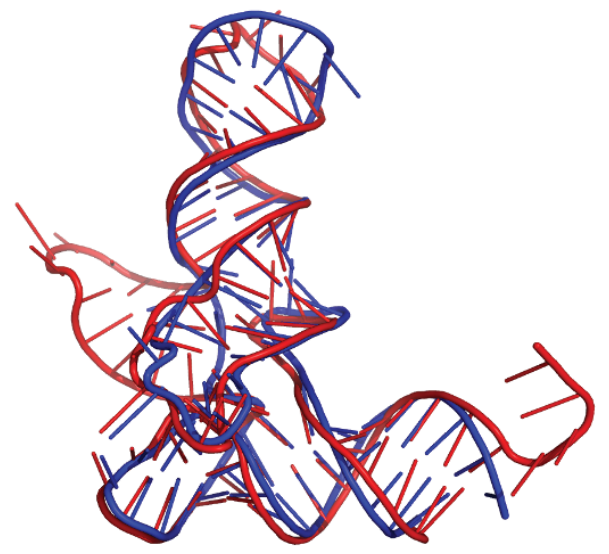

Aligned nucleotides:

65

Sequence Identity

$1.9 \AA$

Secondary Structure Identity: $\quad 88.5 \%$

Structure Identity:

$87.8 \%$

Sequence - ln ( $p$-value):

10. 2

Secondary structure - ln (p-value): 5.2

Structure - $\ln (p-v a l u e): \quad 7.2$

Mean - In (p-value):
D Thermus thermophilus 235 RNA (3d5b:A)

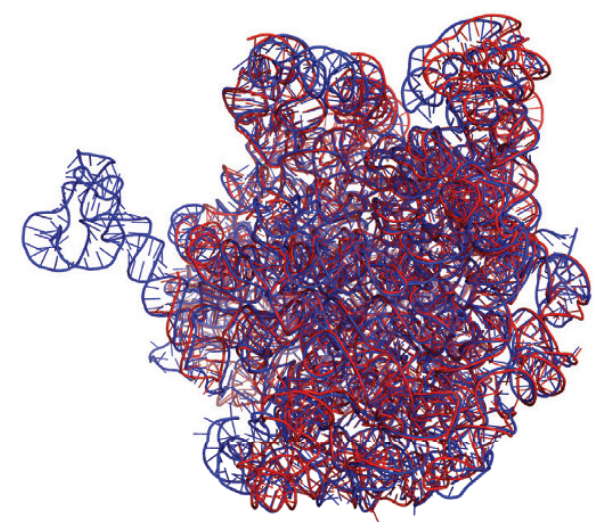

Aligned nucleotides:

2,347

RMSD:

$1.7 \AA$

Sequence Identity:

$52.7 \%$

Secondary Structure Identity: $\quad 75.7 \%$

Structure Identity: $\quad 85.2 \%$

Sequence - ln (p-value): $\quad 37.0$

Secondary structure - ln (p-value): 37.0

Structure - ln (p-value): 37.0

Mean - ln (p-value): $\quad 37.0$

Figure 1 Accurate RNA structure alignments. (A) Staphylococcus phage group I ribozyme (1y0q PDB identifier, chain A) superimposed to a fragment a synthetic construct group I Intron (146b PDB identifier, chain B). (B) tRNA(Leu) of Pyrococcus horikoshii (1wz2 PDB identifier, chain C) superimposed to the tRNA(Met) of Acuifex aeolicus (2ct8 PDB identifier, chain C). (C) Superimposition of two synthetic constructs P4-P6 RNA ribozyme domain (118v and $\underline{2 r 8 s}$ PDB identifiers, chains A and R). (D) 235 ribosomal RNA of Haloarcula marismortui (3cce PDB identifier, chains 0 ) superimposed to the $23 \mathrm{~S}$ ribosomal RNA of Thermus thermophilus ( $\underline{3 \mathrm{~d} 5 \mathrm{~b}}$ PDB identifier, chain A). 


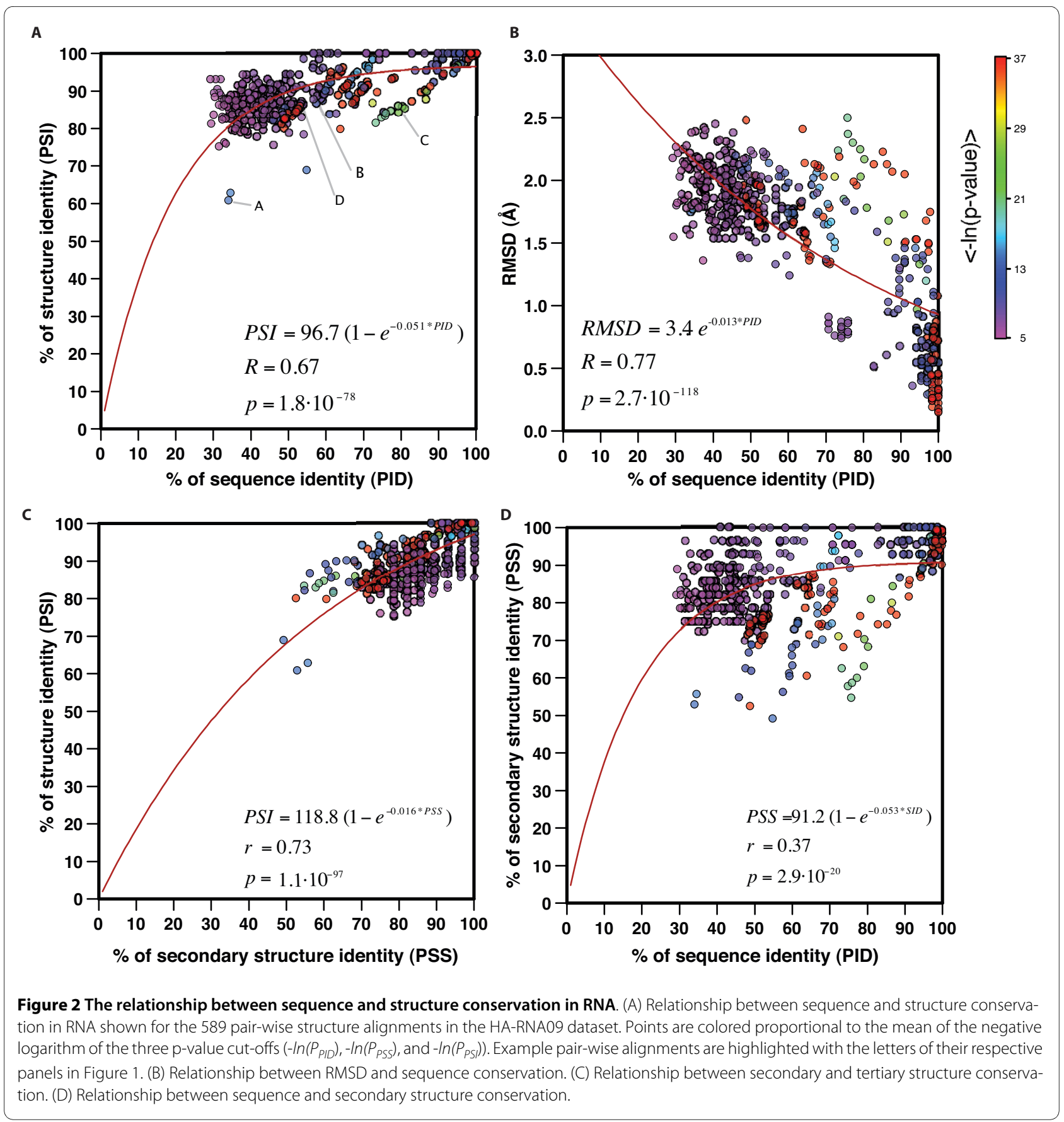

relationship between sequence and structure conservation weakens. Furthermore, to assess the impact of the sequence high identity alignments $(95-100 \%)$ in the fitting of our exponential curves, we removed all pair-wise alignments with sequence identity higher than $95 \%$. The correlation coefficient between RMSD and PID decreased to 0.66 but remained statistically significant $(p$ $=1.5 \cdot 10^{-57}$ ). A similar clustering of the sequence space was previously observed for proteins $[19,50]$, where there was an increase of frequency of alignments with low sequence identity (20-40\%) as well as high sequence identity (90-100\%).

As previously observed, this analysis quantitatively confirmed that RNA secondary structure largely determines tertiary structure. The relationship between secondary structure and tertiary structure conservation in RNA best fitted an exponential decay curve with a correlation coefficient of 0.73 (Figure 2C). The median of secondary structure identity (PSS) for the 589 alignments in the HA-RNA09 set was $85.7 \%$, which agrees with previ- 
ous analysis [34]. However, there was a weaker relationship between sequence and secondary structure conservation in RNA, which could only be fitted to an exponential decay curve with a correlation coefficient of 0.37 (Figure 2D). Pairs of structures that align with relatively high sequence identity could have different secondary structures. For example, the alignment of two group I introns ( $\underline{\mathrm{hr} 2}$ and $\underline{1 \times 8 \mathrm{w}}$ PDB identifiers, chains A) resulted in $75.6 \%$ sequence identity and only $54.7 \%$ secondary structure identity.

\section{A "twilight zone" for RNA sequence alignments}

The 101,475 pair-wise alignments in the RNA09 dataset were divided into three different groups depending on the $-\ln \left(P_{P I D}\right),-\ln \left(P_{P S S}\right)$, and $-\ln \left(P_{P S I}\right)$ cut-offs, which were calculated using the background distribution of similarity scores from a set of 3D structural alignments between RNA molecules with sequence identity lower than $25 \%$ (Methods). The resulting groups included: (i) true positive alignments between related structures with all three cut-offs higher than 4.5, (ii) true negative alignments between dissimilar structures with all three cut-offs lower than or equal to 4.5 , and (iii) medium accuracy alignments with one or two cut-offs higher than 4.5. The sequences of all pair-wise alignments within each group were aligned using the sequence-based alignment program Infernal [51]. This division and alignment allowed us to study of relationship between alignment significance (i.e., Infernal e-value) and the length of the alignment (i.e., shortest sequence between the aligned RNA) as well as to assess the difficulty detecting homology based solely on sequence information. Similar to proteins [19], we observed a "twilight zone" for sequence alignment where true relationship was difficult to assess (Figure 3A). The curve that best separated true positive pairs (green dots) from false positive pairs (orange dots) exponentially decayed from $10^{-10} \mathrm{e}$-value for RNA sequences of $\sim 50$ nucleotides and leveled at $\sim 5 \cdot 10^{-4} \mathrm{e}$-value for RNA larger than 100 nucleotides (Figure 3B). Pair-wise alignments below the "twilight zone" curve included 98,841 true negatives (i.e., unrelated pairs below the curve) and only 152 false positives (i.e., unrelated pairs above the curve). There were a total of 262 RNA structure pairs with all three p-values higher than or equal to 4.5 that were very difficult to detect based on the Infernal alignment score. Those pairs of sequences, which corresponded mainly to tRNA molecules, aligned between 67 and 78 nucleotides with mean sequence identities of $41.7 \%$ and constitute a $23.2 \%$ of false negative rate (i.e., true related pairs below the fitted curve). The analysis of the 262 false negative pair-wise alignments showed that their average PID was much lower (41.7\%) than for the pairs of related structure above the "twilight zone" (71.2\% average PID). However, the secondary structure of those pairs resulted in an $83.1 \%$ average PSS indicating that even though their sequences diverged, their secondary and tertiary structures were conserved. This set thus becomes a very difficult set of related RNA structures to detect by sequence alignment methods.

\section{Discussion}

Our analysis indicate that two related RNA molecules conserve a structure core of at least $\sim 50$ nucleotides, which can be superimposed within $4.0 \AA$ RMSD. Such conserved structure core starts diverging as sequence identity decreases below $50 \%$ and becomes noteworthy (i.e., structural divergence $>20 \%$ ) for pairs of RNA structures that superimpose with sequence identity below $40 \%$. Moreover, the exact relationship between sequence and structure conservation for pairs of distant RNA molecules (that is, resulting in 30 to $60 \%$ sequence identity alignments) is less evident, which results in a correlation coefficient of 0.34. Homologous pairs of RNA molecules will diverge into different structures when there is a significant decrease in the identity of their sequences. Therefore, it is more difficult to assess structure conservation based on sequence diversity in the low regime of sequence identity (i.e, <60\%). Highly similar structures conserve their base pairing. The degree of conservation between tertiary and secondary structure in RNA results in a correlation of 0.73 . However, the relationship between sequence and secondary structure conservation is weakly correlated, which agrees with the difficulty of predicting secondary structure from RNA sequence alone. Similar conclusions were obtained with the ARTS program, which used a $>90 \%$ secondary structure identity threshold for structural classification of RNA [34]. Our results show that for conserved RNA structure cores, high secondary structure identity implies high tertiary structure identity but not necessarily high sequence identity. This reflects the co-variation effect in RNA that requires balancing a single mutation with a second change in the based-paired nucleotide to maintain its secondary and tertiary structures.

To accurately detect a pair of related RNA structures from sequence, their alignment should result in Infernal e-values smaller than $5 \cdot 10^{-4}$. This result indicate that for RNA, likewise for proteins [19], there is a "twilight zone" for the practical application of homology-based approaches for RNA structure prediction. Using the Infernal program with an e-value cut-off of $5 \cdot 10^{-4}$, we identified 50,523 pair-wise alignments between RNA sequences from the RFam database [52] and known RNA structures. This represents $26.2 \%$ and $4.5 \%$ coverage of all sequences and families in the RFam database, respectively. Of those, $90.7 \%(45,812)$ were between two sequences that result in alignments above the "twilight zone" curve and represent a set of query sequences for 


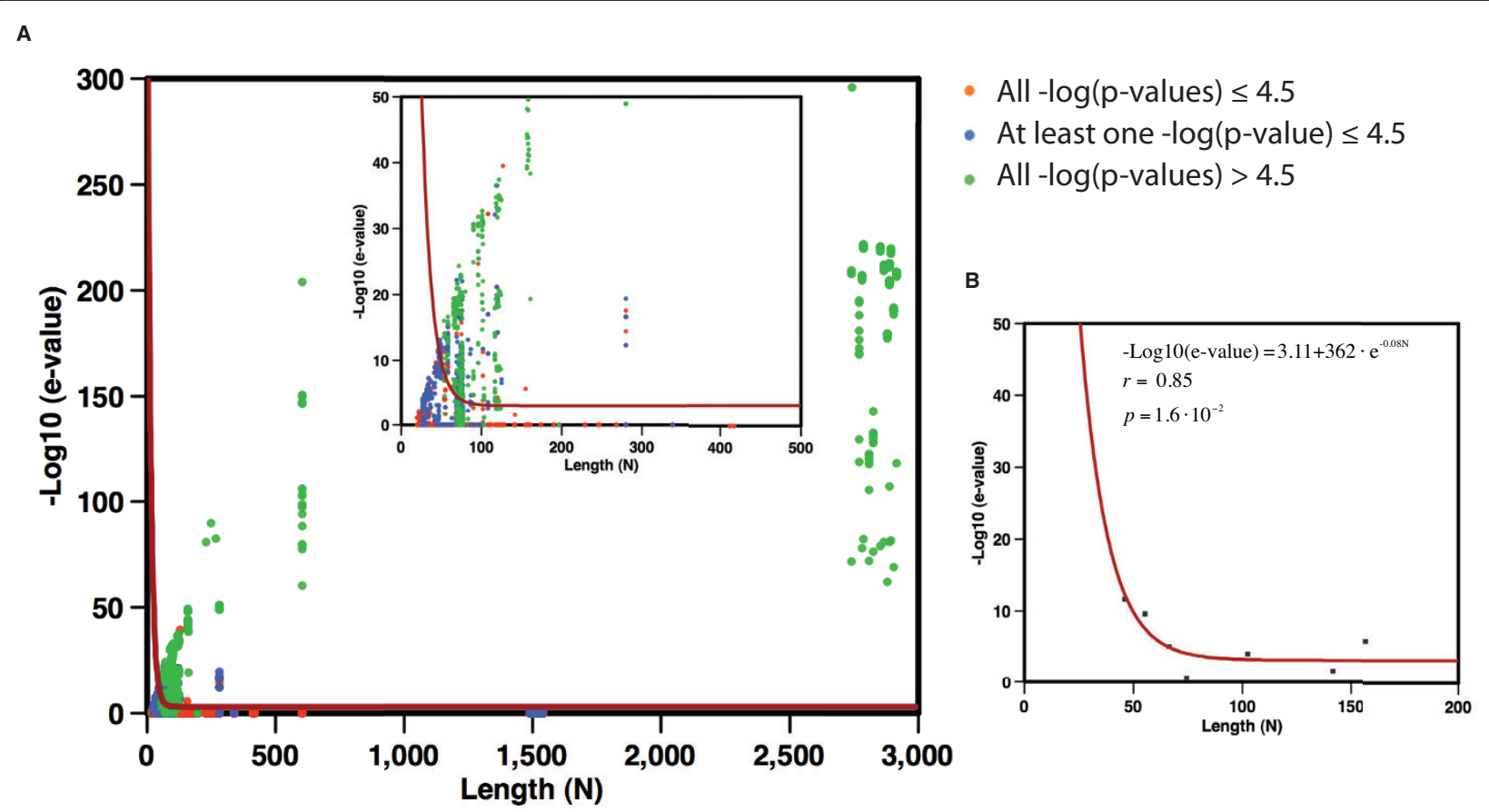

Figure 3 "Twilight zone" for RNA sequence alignments. The length of the shorter of the two aligned sequences $(N)$ is plotted against the e-value of aligning both sequences with the Infernal program. (A) Result from an all-against-all sequence comparison of the RNA sequences in the RNA09 dataset. Green dots correspond to true positive relationships (i.e., with $-\ln \left(P_{P I D}\right),-\ln \left(P_{P S S}\right)$, and $-\ln \left(P_{P S}\right)$ higher than 4.5). Blue dots correspond to medium accuracy alignments (i.e., with at least one of the three scores below or equal to 4.5). Orange dots correspond to true negatives relationships (i.e., with $-\ln \left(P_{P I D}\right),-\ln \left(P_{P S S}\right)$, and $-\ln \left(P_{P S}\right)$ lower or equal to 4.5). The inner plot shows the results for alignments with $N$ shorter than 500 nucleotides. (B) The bestfit curve (red line in panels A and B) that optimally separates true positive from true negative. Matthew's correlation coefficient of 0.81 (-Log 10 (e-value) $=3.11+3.62 \mathrm{e}+02 \cdot \mathrm{e}^{-0.0798 \mathrm{~N}}$, fitting correlation coefficient 0.85 , probability $1.6 \cdot 10^{-2}$ ).

which comparative RNA structure prediction could reliably be used.

It is important to note that our study is currently affected by two circumstances. First, the distribution of RNA structures deposited in the PDB is rather scattered. It is known that the RNA structures in the PDB do not evenly represent the entire RNA structure space. To minimize such problem, we have used a pre-selected dataset of non-identical RNA structures (identity cut-off of $100 \%)$ as well as removing alignments between a structure and its sub-structures. Moreover, such problem will become less relevant with time given the increased pace of deposition of new RNA structures in the PDB. Second, RNA motif comparison has classically been centered on small RNA fragments of about 10 to 30 nucleotides. However, given the intrinsic difficulty of discerning significant alignments from random pairs of short RNA structures or motifs, our work has focused on identifying structural cores of at least $\sim 50$ nucleotides.

\section{Conclusions}

Despite the increasing interest on RNA function and the accelerated deposition of RNA structures, there was a gap between the common knowledge and a quantitative anal- ysis of the relationship between sequence and structure conservation in RNA. Here we have addressed this knowledge gap by applying our RNA alignment method [41] to a set of 451 non-identical RNA structures. The relationship we quantified confirms previous studies in ribosomal RNA $[25,27,28,53]$ and could prove useful to assess whether a particular alignment could be reliably used for comparative RNA structure prediction. We have quantitatively shown that: (i) there is an exponential decay relationship between sequence and structure conservation, (ii) evolution tends to conserve more structure than sequence, and (iii) there is a "twilight zone" for RNA homology detection.

Our study provides an initial assessment of the current limits of comparative modeling of RNA structures. We anticipate that our work will aid the development of new methods for RNA structure prediction from sequence. In the near future, it is expected that large-scale comparative modeling of RNA structures will extend opportunities for answering key questions about RNA evolution, such as the origin of RNA as functional molecules [54]. We have estimated that it would be possible to model by comparative modeling segments of approximately one quarter of all RNA sequences in the RFam database. More 
accurate RNA sequence alignment methods, including those that explicitly use base-pairing information, will be needed to increase the coverage, diversity and accuracy of reliable comparative RNA 3D structure models. Assuming the current growth rate in the number of known RNA structures, comparative modeling will be applicable to a significant number of RNA families within the next few years and thus could play an important bridging role in understanding the atomic mechanisms of RNA folding.

\section{Methods}

Two types of RNA alignments were obtained in our experiment. First, 3D structure-based alignments, which were produced by the SARA program [41] and used to characterize the relationship between sequence and secondary/tertiary structure conservation in RNA. Second, sequence-based alignments, which were produced by the Infernal program [51] and used to characterize a "twilight zone" for RNA homology detection.

\section{Structure-based RNA alignments}

Pairs of RNA structures were aligned using the SARA program, which is based on the unit vector approach [55]. A similar approach was previously used for protein structure alignment by the MAMMOTH program [56]. Briefly, SARA alignments were built by the following procedure: (i) given a RNA structure with $N$ nucleotides, for each $i=1, \ldots, N-1$ extract the vector from the $i$-th to the $(i+1)$-st selected atoms; (ii) normalize all vectors to a unit-distance, and place the tails of all normalized vectors at the origin of a unit-sphere; the resulting collection of normalized vectors is the unit-vector representation of the input RNA. In this work, SARA aligned two RNA structures by selecting only C3' atoms involved in basepairing as computed by the 3DNA program [57]; (iii) calculate the Unit-vector RMS distance between two sets of unit vectors as a score for local RNA structural comparison. This is equivalent to the root mean square deviation (RMSD) between their corresponding normalized vectors after determining the rotation to minimize the RMSD [58]; (iv) the comparison of consecutive unit-spheres generates an all-against-all similarity scoring matrix, which is used in a dynamic programming procedure for the global alignment of two RNA structures using zero end gap penalties [59]; (v) the output alignment is then refined by maximizing the number of equivalent atoms or basepairs within $3.5 \AA$ RMSD using a variant of the MaxSub algorithm, which ensures that the best local alignment is contained in the resulting alignment [56,60]; and (vi) three $\mathrm{p}$-value and their negative logarithms are calculated to assess the statistical significance of the resulting alignment scores.

The three alignment scores calculated by SARA are: first, percentage structure identity (PSI):

$$
P S I=100 \frac{n_{a l}}{N}
$$

where $n_{a l}$ is the number of aligned C3' atoms within a threshold distance of $4.0 \AA$ and $N$ is the length of the shortest of the two RNA structures. Second, percentage of aligned secondary structure (PSS):

$$
P S S=100 \frac{p_{a l}}{N P}
$$

where $p_{a l}$ is the number of aligned base pairs within a threshold distance of $4.0 \AA$ and $N P$ is the smallest number of base pairs of the two aligned RNA structures. Two base pairs are aligned when both $\mathrm{C} 3$ ' atoms of the interacting nucleotides in the first structure are below $4.0 \AA$ to the two C3' of the interacting nucleotides in the second structure. Third, percentage of sequence identity (PID):

$$
P I D=100 \frac{n_{i d}}{N}
$$

where $n_{i d}$ is the number of identical nucleotide types aligned in the structural alignment and $N$ is the length of the shortest of the two RNA structures.

After an alignment is produced, SARA calculates the PSI, PSS and PID scores as well as their associated p-values for estimating the probability of obtaining an equalor better-scored alignment by chance. The distribution of the accuracy scores (i.e., PSI, PSS and PID) for alignments between unrelated RNA structures follows an extreme value distribution and the probability for a given alignment to obtain a score $x$ larger than $z$ can be calculated by integrating the Gumbel distribution:

$$
P(x>z)=1-\exp \left(-\exp \left(-\frac{\pi}{\sqrt{6}} z-\gamma\right)\right)
$$

where $\gamma=0.5772$ and $z$ is

$$
z=\frac{x-\mu}{\sigma}
$$

where $\mu, \sigma$ are the values that best fit the extreme value distribution (Eqn. 4 and Additional file 1 Figure S1).

\section{Sequence-based RNA alignments}

To assess the limits of RNA homology detection, we used the Infernal program [51] to generate a set of covariance models (CMs) for each structure in the RNA09 dataset. The CMs were built using known RNA secondary structures calculated by the 3DNA program [57] from the 3D 
coordinates of the structures. An arbitrary random sequence length of $4 \mathrm{Mb}$ was set to calibrate the local covariance model, which exceed the length of largest ribosomal RNA sequences in the RNA09 dataset $(\sim 2900$ $\mathrm{nt})$. We then performed a leave-one-out procedure removing from the RNA09 dataset one RNA entry at the time and treating it as target sequence of unknown structure. Each target was aligned with Infernal against the remaining set of CMs of known structures or templates. The size of the search space was set to $200 \mathrm{Mb}$ during the search step. All other parameters in Infernal were set at their default values. The searching by Infernal returned a list of hits of each target sequence and the e-value of the statistical significance of the alignments. Infernal resulted in 2,335 top hits with e-value lower than 1.0, which where stored and used to calculate a "twilight zone" for RNA homology detection. Additionally, all the 451 covariance models generated from the RNA structures in RNA09 dataset where used to search homologous RNA sequences in the RFam database using the same Infernalbased protocol. Such analysis allowed us to assess the likely impact of comparative RNA structure prediction.

\section{Alignments datasets}

As of March 2009, the PDB database stored a total of 1,534 structure files containing at least one RNA chain. This initial dataset of structures was filtered by removing RNA structures that: (i) were less than 20 nucleotides long and had less than 4 base pairs; and (ii) were 100\% identical in sequence to another RNA structure in the dataset. The final dataset (RNA09) included 451 RNA chains from 417 PDB entries (Table 1 and Additional file 2). Next, an all-against-all comparison of the structures in the RNA09 set using the SARA program [41] was carried out and resulted in 101,475 pair-wise RNA structure alignments, which were used to create two other datasets: (i) the non-related structure alignments dataset (NRRNA09), which included 50,995 pair-wise alignments (Table 1 and Additional file 3 ) and (ii) the highly accurate structure alignments dataset (HA-RNA09), which included 589 pair-wise alignments (Table 1 and Additional file 4). The NR-RNA09 dataset was generated by selecting only pair-wise alignments in the RNA09 dataset with sequence identity below $25 \%$ and was used for calculating the background distribution of identity scores (Additional file 1 Figure S1). The HA-RNA09 dataset was

Table 1: RNA structure and alignments datasets.

\begin{tabular}{lll}
\hline Dataset & Number of structures & Number of alignments \\
\hline RNA09 & 451 & 101,475 \\
NR-RNA09 & 451 & 50,995 \\
HA-RNA09 & 114 & 589 \\
\hline
\end{tabular}

generated by selecting high-scored alignments (i.e., with all three negative logarithm of p-value $-\ln \left(P_{P S I}\right),-\ln \left(P_{P S S}\right)$, and $-\ln \left(P_{P I D}\right)$ higher than or equal to 4.5$)$ with a crystallographic resolution higher than $5 \AA$ and removing all alignments between a RNA structure and its sub-structures. The ability of the negative logarithm p-values scores for identifying biologically relevant alignments was previously tested by means of functional annotation of the SCOR database [31,38]. The HA-RNA09 dataset, which was generated after applying this triple cut-off to the RNA09 dataset and removing sub-structures, represented a set of related non-identical high-resolution RNA structures. Therefore, the HA-RNA09 dataset is a good compromise between the need of a large set of alignments and the appropriate sampling of the sequence and structure spaces. The entire datasets of RNA structures and alignments used in this work are available as Additional files as well as for downloading at http://sgu.bioinfo.cipf.es/datasets.

\section{Additional material}

Additional file 1 P-value parameter optimization. Fitting of the $\mu$ and $\sigma$ values for the calculation p-values for PID, PSS and PSI.

Additional file 2 RNA09 dataset. Structural alignments in the NR-RNA09 dataset used in the all-against-all structural comparison.

Additional file 3 NR-RNA09 dataset. Structural alignments in the NRRNA09 dataset used for the calculation of the background distributions for PID PSS and PSI.

Additional file 4 HA-RNA09 dataset. Structural alignments in the HARNA09 dataset used to quantify the relationship between sequence and structure in RNA.

\section{Authors' contributions}

EC carried out the computational analysis. EC and MAM-R conceived and designed the study as well as drafted the manuscript. All authors read and approved the final manuscript.

\section{Acknowledgements}

We are very grateful to Dr. Baldo Oliva for fruitful discussions. MAM-R acknowledges support from the Marie Curie International Reintegration program (FP6039722), the Generalitat Valenciana (GV/2007/065), and the Spanish Ministerio de Ciencia e Innovación (BIO2007/66670). EC acknowledges support from the Marie Curie International Outgoing Fellowship program (PIOF-GA-2009237225).

\section{Author Details}

1Structural Genomics Unit, Bioinformatics and Genomics Department, Centro de Investigación Príncipe Felipe, Valencia, Spain and 2Current Address: The Helix Group, Department of Bioengineering, Stanford University, Stanford, CA 94305-5444, USA

Received: 14 December 2009 Accepted: 15 June 2010

Published: 15 June 2010

\section{References}

1. Guerrier-Takada C, Gardiner K, Marsh T, Pace N, Altman S: The RNA moiety of ribonuclease $P$ is the catalytic subunit of the enzyme. Cell 1983, 35(3 Pt 2):849-857.

2. Kruger K, Grabowski PJ, Zaug AJ, Sands J, Gottschling DE, Cech TR: Selfsplicing RNA: autoexcision and autocyclization of the ribosomal RNA intervening sequence of Tetrahymena. Cell 1982, 31(1):147-157. 
3. Doudna JA, Cech TR: The chemical repertoire of natural ribozymes. Nature 2002, 418(6894):222-228.

4. Staple DW, Butcher SE: Pseudoknots: RNA structures with diverse functions. PLOS Biol 2005, 3(6):e213.

5. Onoa B, Tinoco I: RNA folding and unfolding. Curr Opin Struct Biol 2004 14(3):374-379.

6. Sosnick TR, Pan T: RNA folding: models and perspectives. Curr Opin Struct Biol 2003, 13(3):309-316.

7. Westhof E, Massire C: Structural biology. Evolution of RNA architecture. Science 2004, 306(5693):62-63.

8. Cruz JA, Westhof E: The dynamic landscapes of RNA architecture. Cell 2009, 136(4):604-609.

9. Berman $\mathrm{H}$, Henrick K, Nakamura $\mathrm{H}$, Markley JL: The worldwide Protein Data Bank (wwPDB): ensuring a single, uniform archive of PDB data. Nucleic Acids Res 2007:D301-303.

10. Capriotti E, Marti-Renom MA: Computational RNA structure prediction Current Bioinformatics 2008, 3:32-45.

11. Marti-Renom MA, Stuart AC, Fiser A, Sanchez R, Melo F, Sali A: Comparative protein structure modeling of genes and genomes. Annu Rev Biophys Biomol Struct 2000, 29:291-325.

12. Lesk AM, Chothia C: How different amino acid sequences determine similar protein structures: the structure and evolutionary dynamics of the globins. J Mol Biol 1980, 136(3):225-270.

13. Chothia C, Lesk AM: The evolution of protein structures. Cold Spring Harb Symp Quant Biol 1987, 52:399-405.

14. Chothia C, Lesk AM: The relation between the divergence of sequence and structure in proteins. Embo $\mathrm{J} 1986,5(4): 823-826$.

15. Godzik A, Jambon M, Friedberg I: Computational protein function prediction: Are we making progress? Cell Mol Life Sci 2007.

16. Holm L, Sander C: Dali: a network tool for protein structure comparison. Trends Biochem Sci 1995, 20(11):478-480.

17. Murzin AG, Brenner SE, Hubbard T, Chothia C: SCOP: a structural classification of proteins database for the investigation of sequences and structures. J Mol Biol 1995, 247:536-540.

18. Orengo CA, Michie AD, Jones S, Jones DT, Swindells MB, Thornton JM: CATH--a hierarchic classification of protein domain structures. Structure 1997, 5:1093-1108.

19. Rost B: Twilight zone of protein sequence alignments. Protein Eng 1999, 12(2):85-94

20. Sali A, Overington JP: Derivation of rules for comparative protein modeling from a database of protein structure alignments. Protein $\mathrm{SC}$ 1994, 3(9):1582-1596.

21. Levitt M: Detailed molecular model for transfer ribonucleic acid. Nature 1969, 224(5221):759-763.

22. Yusupov MM, Yusupova GZ, Baucom A, Lieberman K, Earnest TN, Cate JH, Noller HF: Crystal structure of the ribosome at $5.5 \mathrm{~A}$ resolution. Science 2001, 292(5518):883-896.

23. Wimberly BT, Brodersen DE, WM J, Morgan-Warren RJ, Carter AP, Vonrhein C, Hartsch T, Ramakrishnan V: Structure of the $30 \mathrm{~S}$ ribosomal subunit. Nature 2000, 407(6802):327-339.

24. Ban N, Nissen P, Hansen J, Moore PB, Steitz TA: The complete atomic structure of the large ribosomal subunit at $2.4 \mathrm{~A}$ resolution. Science 2000, 289(5481):905-920.

25. Noller HF: RNA structure: reading the ribosome. Science 2005, 309(5740):1508-1514

26. Smith TF, Lee JC, Gutell RR, Hartman H: The origin and evolution of the ribosome. Biol Direct 2008, 3:16

27. Caetano-Anolles G: Tracing the evolution of RNA structure in ribosomes. Nucleic Acids Res 2002, 30(11):2575-2587.

28. Tamura M, Holbrook SR: Sequence and structural conservation in RNA ribose zippers. J Mol Biol 2002, 320(3):455-474.

29. Wuyts J, Van de Peer Y, De Wachter R: Distribution of substitution rates and location of insertion sites in the tertiary structure of ribosomal RNA. Nucleic Acids Res 2001, 29(24):5017-5028.

30. Griffiths-Jones S, Moxon S, Marshall M, Khanna A, Eddy SR, Bateman A: Rfam: annotating non-coding RNAs in complete genomes. Nucleic Acids Res 2005:D121-124

31. Tamura M, Hendrix DK, Klosterman PS, Schimmelman NR, Brenner SE, Holbrook SR: SCOR: Structural Classification of RNA, version 2.0. Nucleic Acids Res 2004:D182-184.
32. Leontis NB, Westhof E: Analysis of RNA motifs. Curr Opin Struct Bio/ 2003, 13(3):300-308

33. Murthy VL, Rose GD: RNABase: an annotated database of RNA structures. Nucleic Acids Res 2003, 31(1):502-504.

34. Abraham M, Dror O, Nussinov R, Wolfson $\mathrm{HJ}$ : Analysis and classification of RNA tertiary structures. RNA 2008, 14(11):2274-2289.

35. Murray $\mathrm{L}$, Arendall WB, Richardson DC, Richardson JS: RNA backbone is rotameric. Proc Natl Acad Sci USA 2003, 100(24):13904-13909.

36. Holbrook SR: Structural principles from large RNAs. Annu Rev Biophys 2008, 37:445-464.

37. Wadley LM, Keating KS, Duarte CM, Pyle AM: Evaluating and learning from RNA pseudotorsional space: quantitative validation of a reduced representation for RNA structure. J Mol Biol 2007, 372(4):942-957.

38. Capriotti E, Marti-Renom MA: SARA: a server for function annotation of RNA structures. Nucleic Acids Res 2009:W260-265.

39. Dror O, Nussinov R, Wolfson H: ARTS: alignment of RNA tertiary structures. Bioinformatics 2005, 21(Suppl 2):ii47-ii53.

40. Ferre F, Ponty Y, Lorenz WA, Clote P: DIAL: a web server for the pairwise alignment of two RNA three-dimensional structures using nucleotide, dihedral angle and base-pairing similarities. Nucleic Acids Res 2007:W659-668.

41. Capriotti E, Marti-Renom MA: RNA structure alignment by a unit-vector approach. Bioinformatics 2008, 24(16):i112-118.

42. Das R, Baker D: Automated de novo prediction of native-like RNA tertiary structures. Proc Natl Acad Sci USA 2007, 104(37):14664-14669.

43. Parisien M, Major F: The MC-Fold and MC-Sym pipeline infers RNA structure from sequence data. Nature 2008, 452(7183):51-55.

44. Sharma S, Ding F, Dokholyan NV: iFoldRNA: three-dimensional RNA structure prediction and folding. Bioinformatics 2008, 24(17):1951-1952.

45. Jonikas MA, Radmer RJ, Laederach A, Das R, Pearlman S, Herschlag D, Altman RB: Coarse-grained modeling of large RNA molecules with knowledge-based potentials and structural filters. RNA 2009, 15(2):189-199.

46. Peleg O, Brunak S, Trifonov EN, Nevo E, Bolshoy A: RNA secondary structure and squence conservation in $\mathrm{C} 1$ region of human immunodeficiency virus type 1 env gene. AIDS Res Hum Retroviruses 2002, 18(12):867-878.

47. Lisi V, Major F: A comparative analysis of the triloops in all highresolution RNA structures reveals sequence structure relationships. RNA 2007, 13(9):1537-1545.

48. Schudoma C, May P, Nikiforova V, Walther D: Sequence-structure relationships in RNA loops: establishing the basis for loop homology modeling. Nucleic Acids Res 2010, 38(3):970-980.

49. Bradley RK, Pachter L, Holmes I: Specific alignment of structured RNA stochastic grammars and sequence annealing. Bioinformatics 2008, 24(23):2677-2683.

50. Vitkup D, Melamud E, Moult J, Sander C: Completeness in structural genomics. Nat Struct Biol 2001, 8:559-566.

51. Nawrocki EP, Kolbe DL, Eddy SR: Infernal 1.0: inference of RNA alignments. Bioinformatics 2009, 25(10):1335-1337

52. Gardner PP, Daub J, Tate JG, Nawrocki EP, Kolbe DL, Lindgreen S, Wilkinson AC, Finn RD, Griffiths-Jones S, Eddy SR, et al:: Rfam: updates to the RNA families database. Nucleic Acids Res 2009:D136-140.

53. Mears JA, Cannone JJ, Stagg SM, Gutell RR, Agrawal RK, Harvey SC: Modeling a minimal ribosome based on comparative sequence analysis. J Mol Biol 2002, 321(2):215-234.

54. Vaughn M, Martienssen R: It's a Small RNA World, After All. Science 2005, 309(5740):1525-1526

55. Kedem K, Chew LP, Elber R: Unit-vector RMS (URMS) as a tool to analyze molecular dynamics trajectories. Proteins 1999, 37(4):554-564.

56. Ortiz AR, Strauss CE, Olmea O: MAMMOTH (matching molecular models obtained from theory): an automated method for model comparison. Protein Sci 2002, 11(11):2606-2621.

57. Lu XJ, Olson WK: 3DNA: a software package for the analysis, rebuilding and visualization of three-dimensional nucleic acid structures. Nucleic Acids Res 2003, 31(17):5108-5121

58. Chew LP, Huttenlocher D, Kedem K, Kleinberg J: Fast detection of common geometric substructure in proteins. J Comput Biol 1999, 6(34):313-325. 
59. Needleman SB, Wunsch CD: A general method applicable to the search for similarities in the amino acid sequence of two proteins. $\mathrm{J} \mathrm{Mol} \mathrm{Biol}$ 1970, 48:443-453.

60. Siew N, Elofsson A, Rychlewski L, Fischer D: MaxSub: an automated measure for the assessment of protein structure prediction quality. Bioinformatics 2000, 16(9):776-785

doi: 10.1186/1471-2105-11-322

Cite this article as: Capriotti and Marti-Renom, Quantifying the relationship between sequence and three-dimensional structure conservation in RNA BMC Bioinformatics 2010, 11:322

Submit your next manuscript to BioMed Central and take full advantage of:

- Convenient online submission

- Thorough peer review

- No space constraints or color figure charges

- Immediate publication on acceptance

- Inclusion in PubMed, CAS, Scopus and Google Scholar

- Research which is freely available for redistribution

Submit your manuscript at www.biomedcentral.com/submit 